Note

\title{
Synthesis of 1,2-anhydro-D-altropyranose and -D-allopyranose benzyl ethers
}

\author{
Yuguo Du, Wang Mao, Fanzuo Kong * \\ Research Center for Eco-Emironmental Sciences, Academia Sinica, P.0. Box 2871. Beijing 100085, People's \\ Republic of China
}

Received 21 August 1995: accepled in revised form 20 Novamber 1995

Keywords: Benayl ethers; Altropyranose; Allopyranose

1,2-Anhydro-3,4,6-tri- $O$-benzyl- $\beta$-D-altropyranose (11) and 1,2-anhydro-3,4,6-tri- $O$ benzyl- $\alpha$-D-allopyranose (21) are useful monomers for stereoregular polymerization to afford $\alpha-(1 \rightarrow 2)$-linked D-altropyranan or $\beta-(1 \rightarrow 2)$-linked D-allopyranan, which are valuable model compounds for immunological research [1], and may serve as glycosyl donors in the stereospecilic synthesis of oligosaccharides [2] and other biomedical products [3]. According to the literature [4], altrose and allose derivatives occur frequently as components of biouctive natural products. For example. Epipodophyllotoxin altrosides were used as antitumor agents to increase the mean survival time of mice inoculated with P388 leukaemia cells by $220 \%$ [5]. It was also reported that some oligosaccharides containing altropyranose could be used as wound healing agents [6]. and some aryl $\beta$-D-allopyranosides were observed in the leaves of a higher plant (Protea rubropilosa) used in glycosidase-specificity studies [7]. These results have caused us to study the synthesis of the title anhydro sugars via an intramolecular $\mathrm{S}_{\mathrm{N}} 2$ reaction [8] of a $\mathrm{C}-2$ alkoxide with a $\mathrm{C}-1$ bearing a leaving group, or an inverse ring closure [9] reaction of a $\mathrm{C}-1$ alkoxide with a $\mathrm{C}-2$ attached to a leaving group. Thus. methyl 4,6-O-benzylidene- $\alpha-D$-altropyranoside (1) was prepared from D-glucose [10]. and selective 2-O-allylation of 1 [11] gave methyl 2- $O$-allyl-4.6-O-benzylidene- $\alpha$-Daltropyranoside (2) in satisfactory yield (78.7\%). The structure of 2 was established from its 'H NMR spectrum by single frequency decoupling and further confirmed by the preparation of its derivatives $(3,4)$. Carefully controlled acid-catalyzed debenzylicienation of 2 furnished methyl 2-O-allyl- $\alpha-\mathrm{D}$-altropyranoside (5), and benzylation of 5

\footnotetext{
- Corresponding author.
} 
afforded methyl 2-O-allyl-3,4,6-tri- $O$-benzyl- $\alpha$-D-altropyranoside (6) in moderate yield. Hydrolysis $(6 \rightarrow 7)$ followed by deallylation of 7 with $\mathrm{PdCl}_{2}$ afforded 3,4,6-tri- $O$-benzyl-D-altropyranose (8) as a syrup. An alternative preparation of 8 (i.e., benzylation of 2 at $\mathrm{C}-3$ followed by debenzylidenation, then benzylation at $\mathrm{C}-4$ and $\mathrm{C}-6$ ) was not successful as decomposition occurred at the debenzylidenation stage. Acetylation of $\mathbf{8}$ with acetic anhydride in pyridine gave 1,2-di- $O$-acetyl-3,4,6-tri- $O$-benzyl- $\alpha$-D-altropyranose (9) quantitatively, and treatment of 9 with dry hydrogen chloride in diethyl ether furnished the key intermediate, 2-O-acetyl-3,4,6-tri- $O$-benzyl- $\alpha$-D-altropyranosyl chloride (10). On treatment with potassium tert-butoxide in oxolane, 10 was converted to the target compound, 1.2-anhydro-3,4,6-tri- $O$-benzyl- $\beta$-D-altropyranose (11) which was identified by TLC and ${ }^{1} \mathrm{H}$ NMR spectroscopy. The up-field signal at $\delta 3.40$ $\left(J_{\mathrm{H} \cdot 1 \cdot \mathrm{H} \cdot \mathrm{z}}=J_{\mathrm{H} \cdot 2 \cdot \mathrm{H} \cdot 3}=4.0 \mathrm{~Hz}\right)$ is characteristic of the 1,2-epoxide ring [8.9]. Since 11 was not stable, it was not possible to obtain a satisfactory elemental analysis. Further identification was carried out by methanolysis of 11 in absolute $\mathrm{MeOH}$ in the absence of a promoter at room temperature, giving the 1,2-trans linked product, methyl 3,4,6-tri$O$-benzyl- $\alpha$-D-altropyranoside (12), in a quantitative yield.

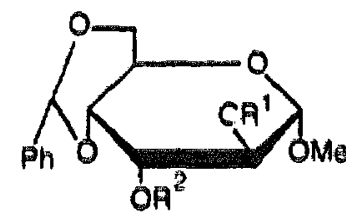

$\begin{array}{cc}R^{1} & R^{2} \\ 1 H & H \\ 2 \text { All } & H \\ 3 \text { All AC } \\ \text { 4a All Bn }\end{array}$

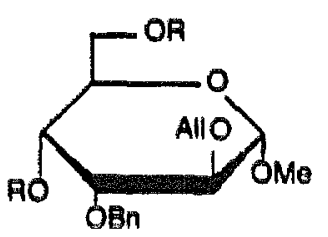

$4 b \mathrm{R}=\mathrm{H}$

Ac R $\mathrm{A}$ AC
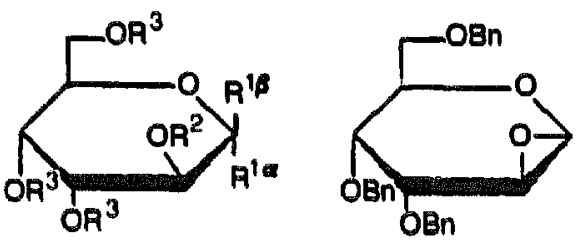

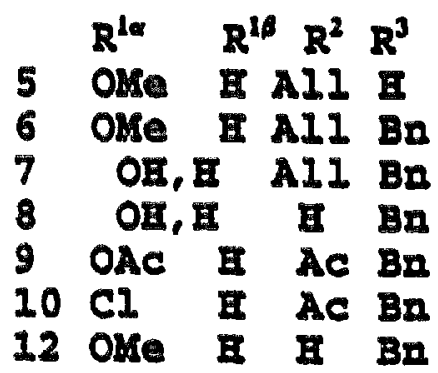

11

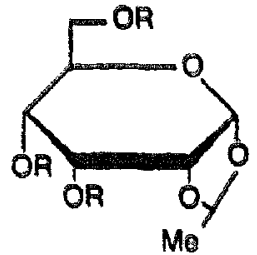

$13 R=A C$ $14 R=B n$

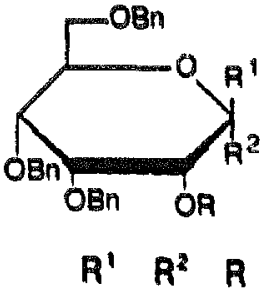

$15 \mathrm{H}, \mathrm{OH} \quad \mathrm{H}$

16 H,OAG AC

$17 \mathrm{H} \mathrm{CI} \mathrm{AC}$

$18 \mathrm{H} F A C$

$22 \mathrm{OMeH} \mathrm{H}$

23 OMe H AC

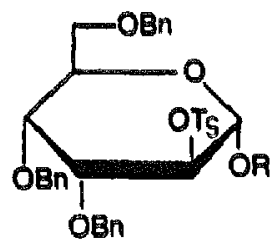

$19 \mathrm{R}=\mathrm{H}$

$20 R=A C$

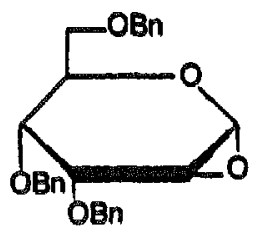

21 
The synthesis of 1,2-anhydro-3,4,6-iti- $O$-benzyl- $\alpha$-D-allopyranose (21) was accomplished by inverse ring closure. The synthesis of $\mathbf{2 1}$ was first attempted via the route used for the synthesis of 1,2-anhydro altrose 11 (i.e., by an intramolecular $S_{\mathrm{N}} 2$ reaction of an $\mathrm{C}-2$ alkoxide with a $\mathrm{C}-1$ bearing a leaving group). Thus, 3,4,6-tri- $O$-acetyl-1,2-O( $R$-ethylidene)- $\alpha$-D-allopyranose (13) containing a trace amount of the $S$ isomer was prepared from D-glucose by reported methods [12,13]. Benzylation of 13 with benzyl chloride and $\mathrm{KOH}$ in toluene under reflux gave 3,4,6-tri-O-benzyl-1,2- $O$-( $R$-ethylidene)$\alpha$-D-allopyranose (14) whose hydrolysis with $M$ sulfuric acid in dioxane afforded 3,4,6-tri-O-benzyl-D-allopyranose (15). Subsequent acetylation of 15 with acetic anhydride in pyridine gave the diacetate 16 as an $\alpha, \beta$ mixture, with the $\beta$ anomer predominating. Quantitative conversion of 16 to 2 - $O$-acetyl-3,4,6-tri- $O$-benzyl- $\alpha$-Dallopyranosyl chloride (17) was readily achieved by treating 16 with dry hydrogen chloride in diethyl ether. To prepare the $\beta$-halide suitable for the intramolecular $\mathrm{S}_{N} 2$ reaction, fluorination of 17 with silver fluoride in $\mathrm{CH}_{3} \mathrm{CN}$-benzene (2:5) was conducted [8]. However, the fluorination gave the $\alpha$-linked fluoride (18) as the sole product in a satisfactory yield, as indicated from its ' $H$ NMR spectrum showing $\mathrm{H}-\mathrm{I}$ at $\delta 5.64$ (dd, $J_{\mathrm{H}-\mathrm{I}, \mathrm{H}-2} 6.0, J_{\mathrm{H}-\mathrm{I}, \mathrm{F}} 54.8 \mathrm{~Hz}$ ), which is similar to the values observed for $\alpha$-fluorides (for 2-O-acetyl-3,4,6-tri-O-benzyl- $\alpha$-D-galactopyranosyl [8b] and 2-O-acetyl-3,4-di-O-benzyl-6-deoxy- $\alpha$-D-glucopyranosyl [8c] fluoride, the chemical shifts of $\mathrm{H}-1$ were $\delta 5.73$ and 5.54. while those of $\mathrm{H}-1$ of $\beta$ anomers were $\delta 5.12$ and 5.17, respectively). Further evidenc? for its $\alpha$ configuration was provided by the resistance of 18 to ring closure under normal ring closure conditions used for glycopyranosyl $\beta$-fluorides $[8 \mathrm{~b}, 8 \mathrm{c}]$. Since this synthesis of the necessary precursor $\beta$-fluoride was unsuccessful, our attention turned to the inverse ring closure [9]. Thus. 3,4.6-tri-O-benzyl-D-altropyranose (8) wass tosylated with $\mathrm{TsCl} .5 \%$ aq $\mathrm{NaOH}$ and $\mathrm{Bu}_{4} \mathrm{NHSO}_{4}$ in $\mathrm{CH}_{2} \mathrm{Cl}_{2}$ (phase transfer conditions). or with $\mathrm{TsCl}$ in pyridine containing $\mathrm{K}_{2} \mathrm{CO}_{3}$ (powder, I equiv) and 4-dimethyliminopyridine (0.1 equiv). 3,4,6-Tri-O-benzyl-2-O-p-10luenesulfonyl- $\alpha$-12-alltropyranose (19) was the sole product (65\%. former method: $53 \%$. latter method). In both cases, the unreacted starting material was recovered and could be reused. The structure of 19 wass confirmed via the acetylated derivative 20 (only the $\alpha$ anomer was obtained). Ring closure of 19 with potassium rert-butoxide in dry oxolane gave the sole product. 1.2-anhydro-3,4,6-tri $O$-benzyl- $\alpha$-1)-allopyranose (21), which was identified by TLC and ${ }^{1} H$ NMR spectroscopy. As observed for 11, the 'H NMR spectrum of 21 gave a characteristic peak at $\delta 3.30\left(J_{\mathrm{H}-1 . \mathrm{H}-2} 2.8, J_{\mathrm{H}-2 . \mathrm{H}-3} 3.4 \mathrm{~Hz}\right)$. Quantitative methanolysis, giving methyl 3,4,6-tri- $O$-benzyl- $\beta$-D-allopyranoside (22), further confirmed the structure of 21. The structure of 22 was verified by acetylation. since the acetylated compound 23 gave a clearer 'H NMR spectrum than 22.

\section{Experimental}

General methods and materials.-See ref. [8a].

Methyl 2-O-ally/-4,6-O-benzylidene- $\alpha$-1)-altropyranoside (2).-To a solution of methyl 4,6-O-benzylidene- $\alpha$-D-altropyranoside [10] (1, 2.03 g, $7.22 \mathrm{mmol})$ in 
dichloromethane $(15 \mathrm{~mL})$ was added tetrabutylammonium iodide $(0.2 \mathrm{~g}, 5.41 \mathrm{mmol})$, $5 \%$ aq sodium hydroxide $(6 \mathrm{~mL})$, and allyl bromide $(1 \mathrm{~mL}, 11.7 \mathrm{mmol})$. The mixture was stirred at room temperature for about $20 \mathrm{~h}$. then diluted with dichloromethane (25 $\mathrm{mL})$ and washed with water $(3 \times 10 \mathrm{~mL})$. The organic layer was dried $\left(\mathrm{Na}_{2} \mathrm{SO}_{4}\right)$ and concentrated to a syrup that was purified by column chromatography with $2: 1$ petroleum ether-EtOAc to give 2 as a syrup $(1.83 \mathrm{~g}, 78.7 \%) ;[\alpha]_{\mathrm{D}}+19.4^{\circ}\left(\mathrm{c} 0.6, \mathrm{CHCl}_{3}\right) ;{ }^{1} \mathrm{H}$ NMR: $\delta$ 7.58-7.35 (m, $5 \mathrm{H}, \mathrm{Ph} H), 6.00-5.89\left(\mathrm{~m}, 1 \mathrm{H}, \mathrm{CH}_{2}=\mathrm{CH}-\mathrm{H}, 5.65(\mathrm{~s}, 1 \mathrm{H}\right.$, PhC H ), 5.40-5.25 (m, $\left.2 \mathrm{H}, J 17.1,1.5,10.3 \mathrm{~Hz}, \mathrm{CH}_{2}=\mathrm{CH}-\right)_{1} 4.74(\mathrm{~s}, 1 \mathrm{H}, \mathrm{H}-1), 4.31$ (dd, $\left.1 \mathrm{H}, J_{5.6} 5.0, J_{6,6^{\prime}} 10.3 \mathrm{~Hz}, \mathrm{H}-6\right), 4.20-4.15(\mathrm{~m}, 2 \mathrm{H}, \mathrm{H}-2,5), 4.13-4.08$ (m, $2 \mathrm{H}$, $\left.\mathrm{CH}_{2}=\mathrm{CH}-\mathrm{C} H_{2}\right), 3.95$ (dd, $\left.1 \mathrm{H}, J_{3,4} 2.7, J_{4,5} 9.8 \mathrm{~Hz}, \mathrm{H}-4\right), 3.85\left(\mathrm{t}, \mathrm{I} \mathrm{H}, J_{5,6^{\prime}} 10.3, J_{6,6^{\prime}}\right.$ $10.3 \mathrm{~Hz}, \mathrm{H}-6^{\prime}$ ), 3.66 (dd, $1 \mathrm{H}, J_{2,3} 1.4, J_{3,4} 2.7 \mathrm{~Hz}, \mathrm{H}-3$ ), $3.49\left(\mathrm{~s}, 3 \mathrm{H}, \mathrm{OC} \mathrm{H}_{3}\right.$ ). Anal. Calcd for $\mathrm{C}_{17} \mathrm{H}_{22} \mathrm{O}_{6}: \mathrm{C}, 63.35 ; \mathrm{H}, 6.83$. Found: $\mathrm{C}, 63.63 ; \mathrm{H}, 6.82$.

Methyl 3-O-acetyl-2-O-ally/-4,6-O-benzylidene- $\alpha$-D-altropyranoside (3). - Acetylation of 2 (100 mg, $0.27 \mathrm{mmol})$ with pyridine $(2 \mathrm{~mL})$ and acetic anhydride $(1.2 \mathrm{~mL})$ at room temperature for $4 \mathrm{~h}$ gave compound 3 in quantitative yield as a syrup; $[\alpha]_{\mathrm{D}}+4.2^{\circ}$ (c 1.9, $\left.\mathrm{CHCl}_{3}\right)$; ${ }^{1} \mathrm{H}$ NMR: $\delta$ 7.50-7.31 (m, $\left.5 \mathrm{H}, \mathrm{Ph} H\right), 6.05-5.80(\mathrm{~m}, \mathrm{I} \mathrm{H}$, $\mathrm{CH}_{2}=\mathrm{CH}$ ) $, 5.60(\mathrm{~s}, 1 \mathrm{H}, \mathrm{PhCH}), 5.44-5.20(\mathrm{~m}, 3 \mathrm{H}, \mathrm{CH}=\mathrm{CH}-\mathrm{H}-3), 4.65(\mathrm{~s}, 1 \mathrm{H}$, $\mathrm{H}-1), 4.40-4.00\left(\mathrm{~m}, 5 \mathrm{H}, \mathrm{H}-2,4,5\right.$, and $\left.\mathrm{CH}_{2}=\mathrm{CH}-\mathrm{CH}_{2}\right), 3.85-3.70\left(\mathrm{~m}, 2 \mathrm{H}, \mathrm{H}-6,6^{\prime}\right)$, 3.41 (s, $3 \mathrm{H}, \mathrm{OC} \mathrm{H}_{3}$ ), $2.11\left(\mathrm{~s}, 3 \mathrm{H}, \mathrm{COCH}_{3}\right.$ ). Anal. Calcd for $\mathrm{C}_{19} \mathrm{H}_{24} \mathrm{O}_{7}: \mathrm{C}, 62.64 ; \mathrm{H}$, 6.59. Found: C, 62.72; H. 6.38.

Methyl 4.6-di-O-acetyl-2-O-allyl-3-O-benzy/- $\alpha$-D-altropyranoside (4c). - Benzylation [8] of compound 2 ( $1.5 \mathrm{~g} .4 .7 \mathrm{mmol})$ with benzyl bromide $(0.9 \mathrm{~mL}, 6.7 \mathrm{mmol})$ and sodium hydride $(80 \%, 500 \mathrm{mg}$. $16.7 \mathrm{mmol}$ ) gave methyl 2-O-allyl-3-O-benzyl-4,6-Obenzylidene- $\alpha$-D)altropyranoside (4a. $1.74 \mathrm{~g}$ ). Debenzylidenation of $4 \mathrm{a}(1.0 \mathrm{~g})$ with $80 \%$ aq acetic acid furnished methyl 2-O-allyl-3-O-benzyl- $\alpha$-D-altropyranoside (4b) in a low yield $(0.39 \mathrm{~g} .50 \%)$. Acetylation of $4 \mathrm{~b}$ with pyridine and acetic anhydride gave $4 \mathrm{c}$ as a

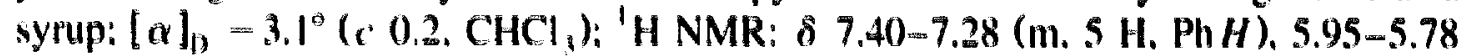
(m. $1 \mathrm{H}, \mathrm{CH}_{2}=\mathrm{CH}$ ), $5.30=5.17$ (m. $2 \mathrm{H}, \mathrm{CH}_{2}=\mathrm{CH}=$ ). 5.09 (dd. I H. $J_{1,4} 2.3 . J_{4.5} 7.9$ Hz, H.4), 4.68 (d, I H, J , 1.5 Hz, H-1), 4.62. 4.56 (2 d. $2 \mathrm{H}, J 9.9 \mathrm{~Hz} . \mathrm{PhCH}$ ). $4.34=4,08(\mathrm{~m}, 3 \mathrm{H}, \mathrm{H}=2.3 .5), 4,06=4,00)\left(\mathrm{m}, 2 \mathrm{H}, \mathrm{CH}_{2}=\mathrm{CH}-\mathrm{CH}_{2}\right), 3.92-3.85(\mathrm{~m}, 2 \mathrm{H}$. $\left.\mathrm{H}-6,6^{\prime}\right), 3.40\left(\mathrm{~s}, 3 \mathrm{H}, \mathrm{OCH} \mathrm{H}_{3}\right), 2,03,2.01(2 \mathrm{~s}, 6 \mathrm{H}, \mathrm{COCH})$.

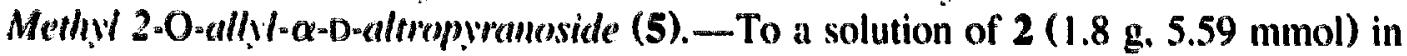
acetone $(2 \mathrm{~mL})$ was added $80 \%$ aq acetic acid $(10 \mathrm{~mL})$. The mixture was stirred under reflux for about $4 \mathrm{~h}$. TLC (1:1 petroleum ether-EtOAc) indicated that the starting material had disappeared. $\mathrm{MeOH}: \mathrm{H}_{2} \mathrm{O}: \mathrm{Et}_{3} \mathrm{~N}(12: 5: 3,8 \mathrm{~mL})$ was added, and the mixture was stirred under reflux for another $30 \mathrm{~min}$. Concentration and purification of the product by column chromatography (1:1 petroleum ether-EtOAc) gave 5 as a syrup $(1.04$ g. $79.5 \%):[\alpha]_{1}+2.4^{\circ}\left(c \quad 0.6, \mathrm{CHCl}_{3}\right):{ }^{1} \mathrm{H}$ NMR: $\delta 6.00-5.80(\mathrm{~m}, 1 \mathrm{H}$, $\mathrm{CH}_{2}=\mathrm{CH}-\mathrm{s}, 5.40-5.20(\mathrm{~m}, 2 \mathrm{H}, \mathrm{CH}=\mathrm{CH}-), 4.75(\mathrm{~s}, \mathrm{I} \mathrm{H}, \mathrm{H} \cdot 1), 4.15-3.65(\mathrm{~m}, 8 \mathrm{H}$. $\mathrm{H}=2,3,4,5,6,6^{\prime}$ and $\mathrm{CH}_{2}=\mathrm{CH}-\mathrm{CH}_{2}$ ). 3.45 (s. $3 \mathrm{H}, \mathrm{OCH}_{3}$ ), 2.90-2.75 (bs, $3 \mathrm{H}, \mathrm{OH}$ ). Anul. Calcd for $\mathrm{C}_{10} \mathrm{H}_{1 \times} \mathrm{O}_{h}:$ C. 51.28: H. 7.69. Found: C, 50.96; H, 7.70.

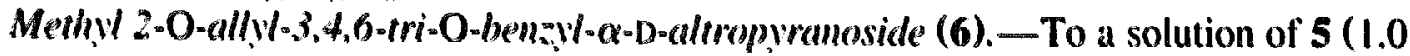
g. $4.27 \mathrm{mmol})$ in anhydrous oxolane $(15 \mathrm{~mL})$ was added, with vigorous stirring in an ice-cold water bath. sodium hydride $(80 \%, 1.25 \mathrm{~g}, 41.7 \mathrm{mmol})$ and benzyl bromide (2.4 g. $14 \mathrm{mmol}$ ). The mixture was stirred and reflux for $8 \mathrm{~h}$. at which time TLC (3:1 
petroleum ether-EtOAc) indicated that the reaction was complete. The mixture was directly subjected to steam distillation to remove the excess benzyl bromide and by-product dibenzyl ether, and then extracted with dichloromethane. The organic layer was concentrated to a syrup that was purified by column chromatography with $4: 1$ petroleum ether-EtOAc to give $6(1.86 \mathrm{~g}, 86.4 \%)$ as a syrup; $[\alpha]_{\mathrm{D}}+2.7^{\circ}(c 4.8$, $\mathrm{CHCl}_{3}$ ); ' $\mathrm{H}$ NMR: $\delta$ 7.40-7.11 (m, $\left.15 \mathrm{H}, \mathrm{Ph} H\right), 5.89-5.70\left(\mathrm{~m}, 1 \mathrm{H}, \mathrm{CH}_{2}=\mathrm{CH}\right.$ ), 5.20-5.05 (m, $\left.2 \mathrm{H}, \mathrm{CH}_{2}=\mathrm{CH}-\right), 4.70(\mathrm{~s}, 1 \mathrm{H}, \mathrm{H}-1), 4.68,4.45(2 \mathrm{~d}, 2 \mathrm{H}, J 10.5 \mathrm{~Hz}$, $\left.\mathrm{PhCH} \mathrm{H}_{2}\right), 4.46,4.59\left(2 \mathrm{~d}, 2 \mathrm{H}, J 10.9 \mathrm{~Hz}, \mathrm{rhCH}_{2}\right), 4.54,4.49(2 \mathrm{~d}, 2 \mathrm{H}, J 11.2 \mathrm{~Hz}$, $\left.\mathrm{PhCH}_{2}\right), 4.30-4.21(\mathrm{~m}, 1 \mathrm{H}, \mathrm{H}-5), 4.00-3.90\left(\mathrm{~m}, 2 \mathrm{H}, \mathrm{CH}_{2}=\mathrm{CH}-\mathrm{CH}_{2}\right), 3.85-3.60$ $\left(\mathrm{m}, 5 \mathrm{H}, \mathrm{H}-2,3,4,6,6^{\prime}\right), 3.40\left(\mathrm{~s}, 3 \mathrm{H}, \mathrm{OC} \mathrm{H}_{3}\right)$. Anal. Calcd for $\mathrm{C}_{31} \mathrm{H}_{36} \mathrm{O}_{6}: \mathrm{C}, 73.81 ; \mathrm{H}$, 7.14. Found: C, 73.79; H, 7.18.

2-O-Allyl-3,4,6-tri-O-benzyl-D-aliropyranose (7). -To a solution of $6(1.8 \mathrm{~g}, 3.57$ mmol) in $60 \%$ aq acetic acid $(15 \mathrm{~mL})$ was added $1 \mathrm{~N}$ hydrochloric acid $(5 \mathrm{~mL})$, and the mixture was stirred at $95^{\circ} \mathrm{C}$ for $8 \mathrm{~h}$, at the end of which time TLC (2:1 petroleum ether-EtOAc) indicated that the starring material had disappeared. The mixture was carefully neutralized with powdered sodium bicarbonate, concentrated and partitioned between water and dichloromethane. The organic layer was dried over sodium sulfate and concentrated. Purification of the residue by column chromatography with 2:1 petroleum ether-EtOAc as solvent furnished syrupy $7(1.32 \mathrm{~g}, 75.4 \%) ;[\alpha]_{D}+2.2^{\circ}(c$ 0.5, $\left.\mathrm{CHCl}_{3}\right)$; ${ }^{1} \mathrm{H}$ NMR: $\delta$ 7.40-7.20 (m, $\left.15 \mathrm{H}, \mathrm{PhH}\right), 5.83-5.68\left(\mathrm{~m}, 1 \mathrm{H}, \mathrm{CH}_{2}=\mathrm{CH}\right)$, 5.20-5.03 (m, $\left.2 \mathrm{H}, \mathrm{CH}_{2}=\mathrm{CH}-\right), 4.82-4.42\left(\mathrm{~m}, 7 \mathrm{H}, \mathrm{H}-1,3 \mathrm{PhC} \mathrm{H}_{2}\right), 4.00-3.84(\mathrm{~m}, 3$ $\left.\mathrm{H}, \mathrm{CH}_{2}=\mathrm{CH}-\mathrm{CH}_{2}, \mathrm{H}-3\right), 3.82-3.75(\mathrm{~m}, 4 \mathrm{H}, \mathrm{H}-2,4,5,6), 3.64-3.59\left(\mathrm{~m}, 1 \mathrm{H}, \mathrm{H}-6^{\prime}\right)$. Anal. Calcd for $\mathrm{C}_{30} \mathrm{H}_{34} \mathrm{O}_{6}$ : C, 73.47; $\mathrm{H}, 6.94$. Found: $\mathrm{C}, 73.14 ; \mathrm{H}, 7.22$.

3,4,6-Tri-O-benzy/-D-altropyranose (8) and 1,2-di-O-acetyl-3,4,6-tri-O-benzyl- $\alpha$-Daltropyranose (9). - To a solution of $7(520 \mathrm{mg}, 1.06 \mathrm{mmol})$ in athydrous methanol (15 $\mathrm{mL}$ ) was added palladium chloride $(20 \mathrm{mg}, 0.11 \mathrm{mmol})$. The mixture was stirred at room temperature for $4 \mathrm{~h}$. Filtration and concentration of the filtrate gave a residue that was purified by column chromatography with $2: 1$ petroleum ether-EtOAc as the solvent furnishing syrupy $8(440 \mathrm{mg} .92 .1 \%) ;[\alpha]_{\mathrm{D}}+4.8^{\ominus}\left({ }^{\circ} 1.9, \mathrm{CHCl}_{3}\right)$. Acetylation of $8(510$ mg. $1.13 \mathrm{mmol})$ with pyridine $(5 \mathrm{~mL})$ and acetic anhydride $(3 \mathrm{~mL})$ at room temperature for 5 h gave compound 9 in quantitative yield as a syrup: $[\alpha]_{\mathrm{D}}+1.2^{\circ}\left(c^{1} 1.2, \mathrm{CHCl}_{3}\right)$; 'H NMR: $\delta 7.40-7.10(\mathrm{~m}, 15 \mathrm{H}, \mathrm{Ph} H), 6.18\left(\mathrm{~d}, 1 \mathrm{H}, J_{1.2} 1.2 \mathrm{~Hz}, \mathrm{H}-1\right), 5.20$ (dd, $1 \mathrm{H}$. $\left.J_{1.2} 1.2, J_{2.3} 2.4 \mathrm{~Hz}, \mathrm{H}-2\right), 4.70-4.40\left(\mathrm{~m}, 6 \mathrm{H}, 3 \mathrm{PhCH} H_{2}\right), 4.25-4.15(\mathrm{~m}, \mid \mathrm{H}, \mathrm{H}-5)$, $3.90-3.70\left(\mathrm{~m}, 4 \mathrm{H}, \mathrm{H}-3,4,6,6^{\prime}\right), 2.15,2.05(2 \mathrm{~s}, 6 \mathrm{H}, \mathrm{COCH})$. Anal. Caled for $\mathrm{C}_{31} \mathrm{H}_{34} \mathrm{O}_{8}: \mathrm{C}, 69.66 ; \mathrm{H}, 6.37$. Found: C, 69.53; H, 6.36.

2-0-Acetyl-3,4,6-tri-O-benzyl- $\alpha$-D-altropyranosyl chloride (10).-A solution of 9 (50 mg, $0.09 \mathrm{mmol})$ in dry diethyl ether $(10 \mathrm{~mL})$ was saturated at $0{ }^{\circ} \mathrm{C}$ with hydrogen chloride gas under a nitrogen atmosphere. The solution was kept at room temperature in a sealed bottle for $2 \mathrm{~h}$, at the end of which time TLC (3:1 petroleum ether-EtOAc) indicated that the reaction was complete. The solution was concentrated under reduced pressure to a syrupy residue which was dissolved in dichloromethane $(1 \mathrm{~mL})$ and concentrated. This procedure was repeated several times to remove the hydrogen chloride. Purification of the product by column chromatography (3:1 petroleum etherEtOAc) gave 10 as a syrup (43 $\mathrm{mg}, 90 \%) ;[\alpha]_{\mathrm{D}}+121^{\circ}\left(\mathrm{c} 2.1, \mathrm{CHCl}_{3}\right) ;{ }^{1} \mathrm{H}$ NMR: $\delta$ 7.40-7.10 (m, $15 \mathrm{H}, \mathrm{Ph} H), 6.01$ (bs, $1 \mathrm{H}, \mathrm{H}-1), 5.34\left(\mathrm{~d}, 1 \mathrm{H}, J_{2.3} 2.9 \mathrm{~Hz}, \mathrm{H}-2\right), 4.86$, 
$4.61\left(2 \mathrm{~d}, 2 \mathrm{H}, J 12.2 \mathrm{~Hz}, \mathrm{PhCH} \mathrm{H}_{2}\right), 4.67,4.53\left(2 \mathrm{~d}, 2 \mathrm{H}, J 12.2 \mathrm{~Hz}, \mathrm{PhCH}_{2}\right), 4.40$, $4.39\left(2 \mathrm{~d}, 2 \mathrm{H}, J 11.0 \mathrm{~Hz}, \mathrm{PhCH}_{2}\right), 3.95-3.70\left(\mathrm{~m}, 5 \mathrm{H}, \mathrm{H}-3,4,5,6,6^{\prime}\right), 2.05$ (s, $3 \mathrm{H}$, $\mathrm{COCH} \mathrm{H}_{3}$ ). Anal. Calcd for $\mathrm{C}_{29} \mathrm{H}_{31} \mathrm{ClO}_{6}$ : C. 68.17; H, 6.07. Found: C, 68.00; H, 6.03.

1,2-An/ydro-3,4,6-tri-O-benzy- $\beta$-D-altropyranose (11). - To a solution of 10 (40 $\mathrm{mg}, 0.078 \mathrm{mmol}$ ) in dry oxolane was added potassium tert-butoxide (12 $\mathrm{mg}, 0.1 \mathrm{mmol})$, and the mixture was stirred at room temperature for $45 \mathrm{~min}$, at the end of which time TLC (3:1 petroleum ether-EtOAc) indicated that the starting material had disappeared. The mixture was concentrated to dryness, and the residue was repeatedly extracted with 3:1 petroleum ether-EtOAc. Concentration of the combined extracts yielded 11 as a syrup (31.5 mg, 93\%); ' $\mathrm{H}$ NMR: $\delta 7.45-7.15(\mathrm{~m}, 15 \mathrm{H}, \mathrm{Ph} H), 4.82-4.44(\mathrm{~m}, 7 \mathrm{H}$, $\mathrm{H}-1$, 3 $\mathrm{PhCH}$ ), 4.30-4.26 (m, I H), 4.00-3.92 (m, I H), 3.88-3.82 (m, I H), $3.80-3.72(\mathrm{~m}, \mid \mathrm{H}), 3.68(\mathrm{~m}, \mid \mathrm{H}), 3.40\left(\mathrm{t}, \mid \mathrm{H}, J_{1,2}=J_{2,3}=4.0 \mathrm{~Hz}, \mathrm{H}-2\right)$.

Methyl 3,4,6-tri-O-benzy/- $\alpha$-D-altropyranoside (12). - Compound 11 (20 mg, 0.046 mmol) was dissolved in anhyd methanol $(2 \mathrm{~mL})$ and kept for $\mathrm{l} \mathrm{h}$ at room temperature. TLC (3:1 petroleum ether-EtOAC) indicated that the reaction was complete. The solution was concentrated to afford 12 quantitatively as a syrup; $[\alpha]_{D}+2.1^{\circ}(c 0.6$, $\mathrm{CHCl}_{3}$ ); ${ }^{1} \mathrm{H}$ NMR: $\delta$ 7.36-7.24 (m. $15 \mathrm{H} . \mathrm{PhH}$ ), 4.62 (d, $1 \mathrm{H}, J 1.23 .7 \mathrm{~Hz}, \mathrm{H}-1$ ), 4.61, 4.46 (2 d. $\left.2 \mathrm{H}, J 10.9 \mathrm{~Hz}_{1} \mathrm{PhCH} \mathrm{H}_{2}\right), 4.60 .4 .58(2 \mathrm{~d}, 2 \mathrm{H}, J \mathrm{Il} .7 \mathrm{~Hz}, \mathrm{PhCH}$ ), 4.53, 4.51 ( 2 d, $2 \mathrm{H}, J \mid 1.1 \mathrm{~Hz}, \mathrm{PhCH}_{2}$ ), 4.24-4.20 (dd, I H. $J_{2,3} 2.6, J_{3,4} 3.1 \mathrm{~Hz}, \mathrm{H}-3$ ), $4.01-3.99$ (dd. I H. J, $J_{1.2}, 7, J_{2,3} 2.6 \mathrm{~Hz}, \mathrm{H}-2$ ), 3.90-3.78 (m, I H. H-5). 3.76 (dd, I H, $J_{3,4}$ 3.1, $\left.J_{4,5} 7.0 \mathrm{~Hz}, \mathrm{H}-4\right), 3.66-3.62\left(\mathrm{~m}, 2 \mathrm{H}, \mathrm{H}-6,6^{\prime}\right), 3.42\left(\mathrm{~s}, 3 \mathrm{H}, \mathrm{OCH} \mathrm{H}_{3}\right)$. Anal. Calce for $\mathrm{C}_{2 \times} \mathrm{H}_{3}, \mathrm{O}_{6} ; \mathrm{C} .72 .41: \mathrm{H}, 6.90$. Found: C, 72.05; H. 7.08.

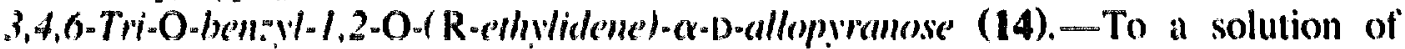

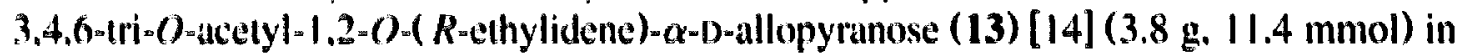
toluent $(30 \mathrm{~mL})$ was added, with vigorous stirring, finely powdered potassium hydroxide ( $12 \mathrm{~g})$. The mixture was boiled under rellux. and benzyl chloride ( $18 \mathrm{~g} .142$ mmol) was added dropwise over a $15 \mathrm{~min}$ period. The mixture was stiered and boiled under rellux for $2 \mathrm{~h}$. at the end al which time TI.C ( $3: 1$ peroleum ether EIOAc) indicated that the reaction was complete. The mixture was directly subjected to stcam distillation to remove the excess benzyl chloride and the by-product dibenzyl ether, and then extracted with dichloromethane. The organic layer was concentrated to a syrup that was purified by column chromatography with $4: 1$ petroleum ether-EtOAc as the eluent to give 14 $(4.9 \mathrm{~g}, 90 \%):[\alpha]_{1}-31^{\circ}\left(\mathrm{c}\right.$ l.1. $\left.\mathrm{CHCl}_{3}\right) ;{ }^{\prime} \mathrm{H}$ NMR: $\delta$ 7.42-7.20 (m, 1.5 H. Ph H ). 5.49 (d. I H. $J_{1.2} 4.0 \mathrm{~Hz}, \mathrm{H}=1$ ). 5.07 (q. I H. J 4.9 Hz, $\mathrm{CHCH}_{3}$ ). 4.88, 4.45 (2 d. $2 \mathrm{H}, J$ 10.6 Hz, PhC $\left.H_{2}\right), 4.78,4.66\left(2 \mathrm{~d}, 2 \mathrm{H}, J 11.7 \mathrm{~Hz} . \mathrm{PhCH}_{2}\right), 4.42,4.34(2 \mathrm{~d} .2 \mathrm{H}, J$ 12.0 Hz. PhCH, ), 4.28-4.22 (m. I H. H-3). 4.06-3.92 (m. 2 H, J, 4.0 Hz, H-2, H-5). 3.68 (dd. I H, $\left.J_{3,4} 2.3, J_{4,5} 7.8 \mathrm{~Hz}, \mathrm{H}-4\right), 3.48$ (dd, I H, $J_{5,6} 2.1, J_{6,6}, 10.6 \mathrm{~Hz}, \mathrm{H}-6$ ). 3,37 (dd. I H. J, J, 3.7. $\left.J_{6,6}, 10.6 \mathrm{~Hz}, \mathrm{H}-6\right), 1.52\left(\mathrm{~d}, 3 \mathrm{H}, J 4.9 \mathrm{~Hz}, \mathrm{CHCH}_{3}\right.$ ). Anal. Calcd for $\mathrm{C}_{24} \mathrm{H}_{32} \mathrm{O}_{12}:$ C. 73.10: H. 6.72. Found: C. 73.28: H. 6.67 .

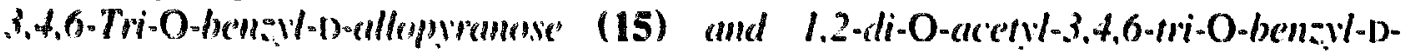
allopyromose (16). - To a solution of $14(2.8 \mathrm{~g} .5 .88 \mathrm{mmol})$ in dioxane $(42 \mathrm{~mL})$ was added $1 \mathrm{M}$ sulfuric acid $(8 \mathrm{~mL})$, and the mixture was boiled under reflux with stirring for $6 \mathrm{~h}$, at the end of which time TLC (2:1 petroleum ether-EtOAc) indicated that the reaction was complete. The mixture was carefully neutralized with powdered sodium bicarbonate, concentrated, and partitioned between water and dichloromethane. The 
organic layer was dried over sodium sulfate and concentrated. Purification of the residue by column chromatography with $2: 1$ petroleum ether-EtOAc as the eluent furnished syrupy $15(2.14 \mathrm{~g}, 81 \%)$ as an $\alpha, \beta$ mixture in a ratio of $3: 2 ;[\alpha]_{\mathrm{D}}-26^{\circ}$ (c 0.9 . $\left.\mathrm{CHCl}_{3}\right) ;{ }^{1} \mathrm{H}$ NMR: $\delta$ 7.40-7.20 (m, $\left.15 \mathrm{H}, \mathrm{Ph} H\right), 5.01\left(\mathrm{~d}, 0.6 \mathrm{H}, J_{1.2} 3.1 \mathrm{~Hz}, \mathrm{H}-1 \alpha\right)$, 4.99-4.42 (m, 6.4 H, $3 \mathrm{PhCH}_{2}, J_{1.2} 8.9 \mathrm{~Hz}, \mathrm{H}-1 \beta$ ), 4.22-4.01 (m, $2 \mathrm{H}, \mathrm{H}-2.3$ ), $3.90-3.58\left(\mathrm{~m}, 4 \mathrm{H}, \mathrm{H}-4,5,6,6^{\prime}\right)$.

Acetylation of $15(1 \mathrm{~g}, 2.22 \mathrm{mmol})$ with pyridine $(5 \mathrm{~mL})$ and acetic anhydride $(3 \mathrm{~mL})$ at room temperature for $4 \mathrm{~h}$ gave compound 16 in a quantitative yield as a syrup consisting of $\alpha$ and $\beta$ anomers in a ratio of $1: 8,[\alpha]_{D}+87^{\circ}$ (mixture, $c 1.1, \mathrm{CHCl}_{3}$ ). For the $\beta$ anomer, ${ }^{1} \mathrm{H}$ NMR: $\delta 7.35-7.20(\mathrm{~m}, 15 \mathrm{H}, \mathrm{Ph} H), 6.08\left(\mathrm{~d}, 1 \mathrm{H}, J_{1.2} 9.6 \mathrm{~Hz}\right.$, $\mathrm{H}-1$ ), 4.77, 4.59 (2 d, $2 \mathrm{H}, J 11.7 \mathrm{~Hz}, \mathrm{PhCH}_{2}$ ), 4.72 (dd, $1 \mathrm{H}, J_{1.2} 9.6, J_{2.3} 2.7 \mathrm{~Hz}$, $\mathrm{H}-2), 4.64,4.47$ (2 d, $2 \mathrm{H}, J 10.9 \mathrm{~Hz}, \mathrm{PhCH}_{2}$ ), 4.57, 4.45 (2 d, $2 \mathrm{H}, J 11.4 \mathrm{~Hz}$, $\left.\mathrm{PhCH} \mathrm{H}_{2}\right), 4.28\left(\mathrm{t}, 1 \mathrm{H}, J_{2.3}=J_{3.4}=2.7 \mathrm{~Hz}, \mathrm{H}-3\right), 4.23-4.13(\mathrm{~m}, 1 \mathrm{H}, \mathrm{H}-5), 3.77$ (dd, ] $\left.\mathrm{H}, J_{3.4} 2.7, J_{4.5} 9.8 \mathrm{~Hz}, \mathrm{H}-4\right), 3.76-3.70\left(\mathrm{~m}, 2 \mathrm{H}, \mathrm{H}-6,6^{\prime}\right), 2.05,1.98(2 \mathrm{~s}, 6 \mathrm{H}$, $\mathrm{COCH}_{3}$ ). Anal. Calcd for $\mathrm{C}_{31} \mathrm{H}_{34} \mathrm{O}_{8}$ (mixture): C, 69.66; H, 6.37. Found: C, 69.57; $\mathrm{H}$, 6.30 .

2-O-Acetyl-3,4,6-tri-O-benzyl- $\alpha$-D-allopyranosyl chloride (17).-The same chlorination and purification conditions $(9 \rightarrow 10)$ were used and compound 17 was obtained as a syrup (249 $\mathrm{mg}, 87 \%$ starting from $300 \mathrm{mg}$ of 16$) ;[\alpha]_{\mathrm{D}}+153^{\circ}\left(c 1.3, \mathrm{CHCl}_{3}\right) ;{ }^{\prime} \mathrm{H}$ NMR: $\delta 7.40-7.20(\mathrm{~m} .15 \mathrm{H}, \mathrm{Ph} H), 6.19\left(\mathrm{~d}, 1 \mathrm{H}, J_{1.2} 4.7 \mathrm{~Hz}, \mathrm{H}-1\right), 4.90-4.86(\mathrm{~m}, 1$ H. H-2), $4.80\left(\mathrm{t}, 2 \mathrm{H}, J 10.0 \mathrm{~Hz}, \mathrm{PhCH}_{2}\right), 4.63-4.42\left(\mathrm{~m}, 5 \mathrm{H}, \mathrm{H}-3,2 \mathrm{PhCH}_{2}\right)$, 4.17-4.10 (m, $2 \mathrm{H}, \mathrm{H}-4.5), 3.90-3.60$ (m, $\left.2 \mathrm{H}, \mathrm{H}-6.6^{\prime}\right), 2.05$ (s, $3 \mathrm{H}, \mathrm{COC} \mathrm{H}_{3}$ ). Anal. Calcd for $\mathrm{C}_{20} \mathrm{H}_{31} \mathrm{ClO}_{6}: \mathrm{C}, 68.17 ; \mathrm{H}, 6.07$. Found: $\mathrm{C}, 67.83 ; \mathrm{H}, 6.17$.

2-O-Acety\%-3,4,6-tri-O-benzy- $\alpha$-D-allopyranosyl fluoride (18). - To a solution of 17 $(350 \mathrm{mg} .0 .69 \mathrm{mmol})$ in $2: 5$ acetonitrile-benzene $(10 \mathrm{~mL})$ was added silver fluoride $(200 \mathrm{mg}, 1.6 \mathrm{mmol})$. The mixture was stirred vigorously for $16 \mathrm{~h}$ in the dark at room temperature, centrifuged, and the lilter cake was washed repeatedly with dichloromethane. The supernatant liquor and combined washings were concentrated. Puritication of the syrup by column chromatography $(3: 1$ petroleum ether-EiOAc) yiclded 18 as a syrup $(287 \mathrm{mg} .85 \%) ;[\alpha]_{\mathrm{p}}+58.1^{\circ}\left(c^{\prime} 0.9, \mathrm{CHCl}_{3}\right) ;{ }^{\prime} \mathrm{H}$ NMR: $\delta$ 7.45-7.20 (m. 15 H. Ph H), 5.64 (dd. 1 H, $\left.J_{1, \mathrm{f}} 54.8, J_{1.2} 6.0 \mathrm{~Hz}, \mathrm{H}-1\right), 4.79,4.50$ (2 d, $\left.2 \mathrm{H}, J / 1.7 \mathrm{~Hz}, \mathrm{PhCH} \mathrm{H}_{2}\right), 4.75-4.70(\mathrm{~m}, 1 \mathrm{H}, \mathrm{H}-2), 4.65-4.53\left(\mathrm{~m}, 5 \mathrm{H}, \mathrm{H}-3,2 \mathrm{PhC} \mathrm{H}_{2}\right)$, 4.30-4.2I (m, $2 \mathrm{H}, \mathrm{H}-4,5), 3.78-3.75\left(\mathrm{~m}, 2 \mathrm{H}, \mathrm{H}-6,6^{\prime}\right), 2.09$ (s, $3 \mathrm{H}, \mathrm{COC} \mathrm{H}_{3}$ ). Anal. Calcd for $\mathrm{C}_{29} \mathrm{H}_{31} \mathrm{FO}_{6}: \mathrm{C}, 70.59 ; \mathrm{H}, 6.28$. Found: $\mathrm{C}, 70.45 ; \mathrm{H}, 6.23$.

3,4,6-Tri-O-benzy/-2-O-p-toluenesulfonyl- $\alpha$-D-altropyranose (19). - To a solution of $8(431 \mathrm{mg}, 0.96 \mathrm{mmol})$ in pyridine $(5 \mathrm{~mL})$ was added $\mathrm{TsCl}(450 \mathrm{mg}, 2.4 \mathrm{mmol})$, 4-dimethylaminopyridine (DMAP, $12.2 \mathrm{mg}, 0.1 \mathrm{mmol})$, and powdered $\mathrm{K}_{2} \mathrm{CO}_{3}(138 \mathrm{mg}$. $1 \mathrm{mmol}$ ). The mixture was stirred at room temperature for $24 \mathrm{~h}$, poured into ice-cold water, and extracted with dichloromethane $(30 \mathrm{~mL})$. The organic layer was washed with cold water $(30 \mathrm{~mL})$ and $1 \mathrm{~N} \mathrm{HCl}(3 \times 20 \mathrm{~mL})$, and dried over $\mathrm{Na}_{2} \mathrm{SO}_{4}$. The solution was concentrated and the resulting residue was purified by column chromatography $(3: 1$ petroleum ether-EIOAc) to give $19(306 \mathrm{mg}, 53 \%)$ as a syrup; $[\alpha]_{\mathrm{D}}+68^{\circ}(\mathrm{c} 0.1) ;{ }^{1} \mathrm{H}$ NMR: $\delta 7.81(\mathrm{~d}, 2 \mathrm{H}, J 7.8 \mathrm{~Hz}, \mathrm{Ph} H$ of Ts$), 7.38-7.12(\mathrm{~m}, 17 \mathrm{H}, \mathrm{Ph} H), 5.51$ (d, $1 \mathrm{H}$, $\left.J_{1.2} 1.6 \mathrm{~Hz}, \mathrm{H}-1\right), 4.76,4.65\left(2 \times 2 \mathrm{~d}, 4 \mathrm{H}, J 12.5 \mathrm{~Hz}, 2 \mathrm{PhC} H_{2}\right), 4.69$ (dd, $1 \mathrm{H}, J_{1.2}$ 1.6, $\left.J_{2,3} 6.0 \mathrm{~Hz}, \mathrm{H}-2\right), 4.56-4.52$ (m, I H, H-3), 4.45 (s, $2 \mathrm{H}, \mathrm{PhCH} \mathrm{H}_{2}$ ), 3.74-3.68 (m, 2 
H. H-5. H-6), 3.64-3.60 (m, $1 \mathrm{H}, \mathrm{H}-4), 3.56$ (dd, $\left.1 \mathrm{H}, J_{5,6} 1, J_{6.6^{\prime}} 9.4 \mathrm{~Hz}, \mathrm{H}-6^{\prime}\right)$. Anal. Calcd for $\mathrm{C}_{34} \mathrm{H}_{36} \mathrm{O}_{8} \mathrm{~S} \cdot 0.5 \mathrm{H}_{2} \mathrm{O}: \mathrm{C}, 66.56 ; \mathrm{H}, 6.04$. Found: C, 66.50; H, 6.13.

I-O-Acetyl-3,4,6-tri-O-benzyl-2-O-p-toluenesulfonyl- $\alpha$-D-altropyranose (20). Acetylation of $19(100 \mathrm{mg}, 0.17 \mathrm{mmol})$ with pyridine $(3 \mathrm{~mL})$ and acetic anhydride (2 $\mathrm{mL})$ at room temperature for $4 \mathrm{~h}$ gave 20 in a quantitative yield as a syrup; $[\alpha]_{\mathrm{D}}-8.6^{\circ}$ (c 0.3): ${ }^{1} \mathrm{H}$ NMR: $\delta 7.69(\mathrm{~d}, 2 \mathrm{H}, J 8.0 \mathrm{~Hz}, \mathrm{Ph} H$ of Ts), 7.40-7.10 (m, $17 \mathrm{H}, \mathrm{Ph} H)$, $6.00\left(\mathrm{~d}, 1 \mathrm{H}, J_{1.2} 1.4 \mathrm{~Hz}, \mathrm{H}-\mathrm{I}\right), 4.64,4.48\left(2 \mathrm{~d}, 2 \mathrm{H}, J 11.8 \mathrm{~Hz}, \mathrm{PhCH} \mathrm{H}_{2}\right), 4.60,4.58$ (2 d, $\left.2 \mathrm{H}, J 11.8 \mathrm{~Hz}, \mathrm{PhCH} H_{2}\right), 4.55$ (dd, $\left.1 \mathrm{H}, J_{1.2} 1.4, J_{2.3} 4.6 \mathrm{~Hz}, \mathrm{H}-2\right), 4.35(\mathrm{~s}, 2 \mathrm{H}$, PhC $H_{2}$ ), 4.16-4.10 (m, I H, H-5), 4.01 (dd, I H, $J_{2.3} 4.6, J_{3,4} 2.9 \mathrm{~Hz}, \mathrm{H}-3$ ), 3.86 (dd, $\left.1 \mathrm{H}, J_{3,4} 2.9, J_{4.5} 9.2 \mathrm{~Hz}, \mathrm{H}-4\right), 3.71-3.66\left(\mathrm{~m}, 2 \mathrm{H}, \mathrm{H}-6,6^{\prime}\right), 2.42\left(\mathrm{~s}, 3 \mathrm{H}, \mathrm{PhC} H_{3}\right), 1.82$ (s. $3 \mathrm{H} . \mathrm{COC} \mathrm{H}_{3}$ ). Anal. Calcd for $\mathrm{C}_{36} \mathrm{H}_{38} \mathrm{O}_{9} \mathrm{~S}: \mathrm{C}, 66.87 ; \mathrm{H}, 5.88$. Found: C. 66.91: H, 5.80 .

1,2-Anhydro-3,4,6-tri-O-benzy- $\alpha$-D-allopyranose (21). - Ring closure of $19(130 \mathrm{mg}$, $0.21 \mathrm{mmol}$ ) under the same conditions used for the preparation of 11 from 10 yielded 21 as a syrup (88 mg, $95 \%)$; ${ }^{i} \mathrm{H}$ NMR: $\delta 7.40-7.15(\mathrm{~m}, 15 \mathrm{H}, \mathrm{Ph} H), 5.00\left(\mathrm{~d}, 1 \mathrm{H}, J_{1.2} 2.8\right.$ Hz. H-1). 4.80, $4.78(2$ d. $2 \mathrm{H}, J 11.7 \mathrm{~Hz}, \mathrm{PhCH}), 4.60,4.52(2 \mathrm{~d} .2 \mathrm{H}, J 10.6 \mathrm{~Hz}$, $\mathrm{PhCH}$ ) $, 4.59,4.55\left(2 \mathrm{~d}, 2 \mathrm{H}, J 10.9 \mathrm{~Hz}, \mathrm{PhCH}{ }_{2}\right), 3.95-3.90(\mathrm{~m}, 2 \mathrm{H}, \mathrm{H}-3,5), 3.70$ (dd. I H. $\left.J_{3,4}, 3.0, J_{4,5} 7.9 \mathrm{~Hz} . \mathrm{H}-4\right), 3.65$ (dd, $1 \mathrm{H}, J_{5,6} 2.5, J_{6,6,} 9.9 \mathrm{~Hz}$ ), 3.60 (dd, $1 \mathrm{H}$, $\left.J_{5,6^{\prime}} 5.1 . J_{6,6^{\prime}} 9.9 \mathrm{~Hz} . \mathrm{H}-6^{\prime}\right), 3.30\left(\mathrm{dd}, 1 \mathrm{H}, J_{1,2} 2.8, J_{2,3} 3.4 \mathrm{~Hz}, \mathrm{H}-2\right), m / z: 432\left(\mathrm{M}^{+}\right.$, $6 \%), 385(20 \%), 342(20 \%), 309(20 \%), 253(60 \%), 203(34 \%), 91$ (basic peak. off scale).

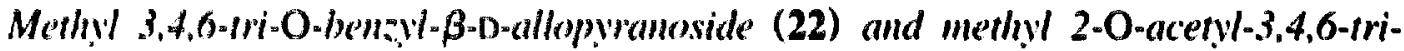

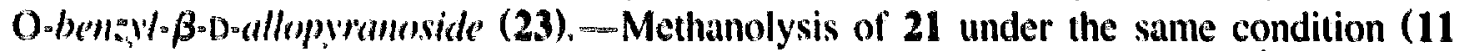
$\rightarrow 12$ ) afforded 22 quantitatively as a syrup: $[\alpha]_{\mathrm{D}}-40.2^{\circ}\left(c 0.5, \mathrm{CHCl}_{3}\right):{ }^{1} \mathrm{H}$ NMR: $\delta$ 7.34-7.24 (111. 10 H. 2 PhoH). 4.68. 4.60 (2 d. 2 H. $\left./ 12.3 \mathrm{~Hz}, \mathrm{PhCH}_{2}\right), 4.63 .4 .46$ (2 d.

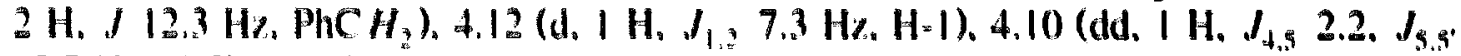
$12.7 \mathrm{~Hz}, \mathrm{H}-5), 3,95$ (dd. I H. $\left.J_{1.2} 7.3, J_{2,3} 9.1 \mathrm{~Hz}, \mathrm{H}-2\right), 3.73-3,69$ (m. I H. H-4), 3.56

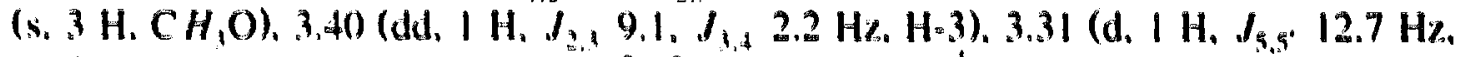
$\left.H^{\circ} \bar{s}^{\prime}\right)$, Acetylution of 22 afforded $23:[\alpha]_{\mathrm{D}}=20^{\circ}(\mathrm{c} 0.7)$; 'H NMR: $\delta$ 7.40-7.10 (m. 15 H. PhH $.4 .85,4.63$ (2 d, $2 \mathrm{H} . J 10.9 \mathrm{~Hz}, \mathrm{PhCH}$ ), 4.81 (d, I H. $J_{1.2} 8.3 \mathrm{~Hz}, \mathrm{H}-1$ ), 4.67 (dd, 1 H. $\left.J_{1,2} 8.3, J_{2,3}, 2.7 \mathrm{~Hz}, \mathrm{H} \cdot 2\right), 4.60\left(\mathrm{~s}, 2 \mathrm{H} . \mathrm{PhCH} \mathrm{H}_{2}\right), 4.58,4.56$ (2 d. $2 \mathrm{H}, J$ $\left.12.1 \mathrm{~Hz}, \mathrm{PhCH} H_{2}\right), 4.30\left(\mathrm{t}, \mathrm{I} \mathrm{H}, J_{2.3}=J_{3,4}=2.7 \mathrm{~Hz}, \mathrm{H}-3\right.$ ). 4.10 (ddd. I H. $J_{4.5} 9.5, J_{5.6}$ 2.0. $J_{5,6^{\prime}} 4.5 \mathrm{~Hz}, \mathrm{H}-5$ ). 3.80 (dd, $\left.1 \mathrm{H}, J_{5, h^{\prime}}, 2.0, J_{6,6^{\prime}} 10.9 \mathrm{~Hz}, \mathrm{H}-6\right), 3.70$ (dd. I H., $J_{5,6^{\circ}}$ 4.5. $\left.J_{6,6^{\prime}} 10.9 \mathrm{~Hz}, \mathrm{H}-6^{\prime}\right), 3.68$ (dd. I H. $\left.J_{3,4}, 2.7, J_{4,5} 9.5 \mathrm{~Hz}, \mathrm{H}-4\right), 3.48(\mathrm{~s}, 3 \mathrm{H}$. $\mathrm{OCH}_{3}$ ) 2.01 (s, $3 \mathrm{H}, \mathrm{COCH} \mathrm{H}_{3}$ ). Anal. Calcd for $\mathrm{C}_{31} \mathrm{H}_{34} \mathrm{O}_{7}:$ C. 71.15: H. 6.72. Found: C. $71.23 ;$ H. 6.70 .

\section{References}

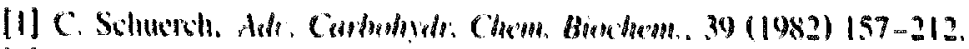

[2] S.J, Dinisheriky, K.F. McClure. J.T. Randelph, and R.B. Ruggeri, Scientce, 260 (1993) 1307-1309.

[3] (a) J.T. Link. S. Raghavan. and S.J. Danishefiky. J. Am. Chem. Soc., 117 (1995) 552-553: (b) J.T. Randolph and S.J. Danishelisty. Anse'u. Chem.. Mn. Ed. Engl. 33 (1994) 1470-1473: (c) A. Delgado and J. Clardy. T. Org. Chem. 58 (1993) 2862-2866.

[4] H.H. Powers, G. Tabakoglu, and H.Z. Silble, Birchem. Prep.. + (1955) 474-503. 
[5] T. Ohnuma. H. Hoshi, H. Kamei, and T. Naito. Ear: Pat. Appl. EP 415.453 (C1.C07H17/04), 6 March 1991.

[6] A.N. Silvetti and A.N. Silvetti. Jr, Eur, Pat. Appl. EP 221.728 (C1.A61K31/70), 13 May 1987.

[7] R.H. Shah and O.P. Bahl, Carbohydr. Res., 65 (1978) 153-158.

[8] (a) G. Yang, F. Kong, and R.R. Fraser, Carbohydr. Res., 258 (1994) 49-58; (b) F. Kong, J. Du, and H. Shang, Carbohydr: Res., 162 (1987) 217-225; (c) G. Yang and F. Kong, J. Carbohydr. Chem., 11 (1992) $595-608$.

[9] (a) Y. Du and F. Kong, Tetrahedron Lett., (1995) 427-430; (b) G. Yang and F. Kong, Carbohydr. Lett., 1 (1994) 137-141.

[10] R.L. Whistler and J.N. BeMiller, Methods Carbohydr. Chem., 8 (1980) 169-170.

[11] P.J. Garegg, T. Iversen, and S. Oscarson, Carbohydr: Res., 50 (1976) C12-C14.

[12] R.L. Whistler and J.N. BeMiller, Methods Carbohydr. Chem., 6 (1972) 123-128.

[13] V.I. Betaneli, M.V. Ovchinikof, L.V. Backinowsky, and N.K. Kochetkov, Carbohỵdr. Res., 107 (1982) $285-291$.

[14] W.E. Dick. Jr, D. Weisleder, and J.E. Hodge, Carbohydr. Res., 42 (1975) 65-72. 